\title{
Transplantation of human adipose stem cell-derived hepatocyte-like cells with restricted localization to liver using acellular amniotic membrane
}

\author{
Jie Yuan ${ }^{1}$, Weihong $\mathrm{Li}^{1}$, Jieqiong Huang ${ }^{1}$, Xinyue Guo ${ }^{1}$, Xueyang $\mathrm{Li}^{1}$, Xin Lu' ${ }^{1}$ Xiaowu Huang ${ }^{2}$ and Haiyan Zhang ${ }^{\text {** }}$
}

\begin{abstract}
Introduction: Adult stem cell-derived hepatocytes transplantation holds considerable promise for future clinical individualized therapy of liver failure or dysfunction. However, the low engraftment of the available hepatocytes in the liver disease microenvironment has been a major obstacle.

Methods: Acellular human amniotic membrane was developed as a three-dimensional scaffold and combined with hepatocyte-like cells derived from human adipose stem cells to engineer a hepatic tissue graft that would allow hepatocyte engraftment in the liver effectively.

Results: The hepatic tissue grafts maintained hepatocyte-specific gene expression and functionality in vitro. When transplanted into the surgical incision in livers for engraftment, the engineered hepatic grafts significantly decreased the degree of liver injury caused by a carbon tetrachloride treatment and generated cords that were similar to the ductal plates in the liver between the acellular human amniotic membrane and the liver of receipts at day 3 post-transplantation. The hepatic tissue grafts maintained the expression of human hepatocyte-specific markers albumin, hepatocyte nuclear factor 4a, and cytochrome P450 2B6 in the liver of receipts, and acquired human-specific drug metabolism ability at eight weeks post-transplantation.

Conclusions: The acellular human amniotic membrane has the ability to maintain the functional phenotype of the hepatocyte-like cells derived from human adipose stem cells. Functional acellular human amniotic membrane-hepatocytes grafts integrated with the liver decreases the acute liver injury of mice. These engineered tissue constructs may support stem cell-based individualized therapy for liver disease and for bioartificial liver establishment.
\end{abstract}

\section{Introduction}

Hepatocyte transplantation, especially using hepatocytes derived from patient adipose stem cells (ASCs), might become safer and easier than whole organ transplantation to cure patients suffering from liver-based metabolic diseases or end-stage liver dysfunction [1-4]. The delivery of these cells and promotion of their efficient engraftment is a challenging task [5]. Placing healthy hepatocytes via intrahepatic injection, intrasplenic delivery, or portal vein infusion

\footnotetext{
* Correspondence: culture@ccmu.edu.cn

'Department of Cell Biology, Municipal Laboratory for Liver Protection and Regulation of Regeneration, Capital Medical University, No. 10, Xitoutiao, You An Men, Beijing 100069, China

Full list of author information is available at the end of the article
}

may hinder engraftment because most diseased livers have altered architectures due to fibrosis and cirrhosis [6]. Therefore, implantation or extrahepatic transplantation to provide an additional site of hepatic function represents a new approach for hepatocyte transplantation [7-9]. New developments in liver engineering technology have motivated research into the development of functional liver grafts that could be connected to a recipient's system $[10,11]$.

An ideal scaffold with relevant aspects of the hepatic microarchitecture and extracellular matrix (ECM) components plays important roles in hepatocyte adhesion and differentiation, as well as in promoting tissue morphogenesis, to create implantable liver tissues or grafts [12-14]. Human amniotic membrane (HAM) has been 
widely used as a graft material for many surgical procedures and for tissue regeneration because this material is inexpensive and easily obtained and because its availability is virtually limitless [15]. Before the material is applied, the amniotic membrane (AM) donor is required to undergo a thorough health screening, and the membrane must undergo an established processing routine, which includes preservation, sterilization, and de-epithelialization [16]. ECM components of acellular human amniotic membrane (AHAM), such as collagen type I, collagen type IV, laminin, and fibronectin, which have biological properties similar to the hepatic ECM, make this membrane a potentially attractive grafting material to facilitate hepatocyte transfer $[17,18]$. Recent findings indicate that HAM may reduce the severity of liver fibrosis in a bile duct ligation rat model $[19,20]$. However, little is known about the possibility of AHAM as a scaffold for hepatocyte attachment, functional maintenance, and transplantation. The aims of this study were to determine the biocompatibility of AHAM with human adipose stem cell-derived hepatocytelike cells (hASC-HLCs) [21] and to assess the ability of AHAM to maintain the function of hepatocytes in vitro and in a hepatic implant in a carbon tetrachloride (CCl4)induced acute hepatic injury mouse model in vivo.

\section{Methods}

\section{Preparation of the AHAM}

HAM was obtained from a cesarean section operation with informed patient consent and under the approval of the Ethics Committee of Capital Medical University (Beijing, China). A maternal donor with no history of premature membrane rupture, endometritis, or meconium ileus was selected at a prenatal visit approximately 2 weeks before delivery and underwent a series of serological tests, including screens for HIV-1/2, hepatitis B, hepatitis $\mathrm{C}$, human $\mathrm{T}$-cell lymphotropic virus type, syphilis, cytomegalovirus, and tuberculosis. Repeat investigations were performed 6 months after delivery.

To prepare the AHAM, the HAM was peeled from the placenta, rinsed extensively in sterile phosphate-buffered saline (PBS) containing $200 \mathrm{U} / \mathrm{mL}$ penicillin, $200 \mathrm{ug} / \mathrm{mL}$ streptomycin, and cut into approximately $5 \mathrm{~cm} \times 5 \mathrm{~cm}$ pieces, which were placed in dishes with the amniotic epithelial layer face up. The HAM pieces were incubated in $0.25 \%$ trypsin with $0.38 \%$ ethylenediamine tetraacetic acid (EDTA; Sigma-Aldrich, St. Louis, MO, USA) for 30 minutes twice at $37^{\circ} \mathrm{C}$, followed by rinsing in sterile PBS and dehydration in glycerol for 48 hours; the glycerol was changed every day. Next, the fresh AHAM pieces were stored at $4{ }^{\circ} \mathrm{C}$ for up to 2 weeks. For generating cryopreserved AHAM, the fresh AHAM pieces were placed in dishes with a 1:1 mixture of glycerol and $0.5 \%$ chondroitin sulfate (Sigma-Aldrich) in MEM-NEAA (Gibco, Carlsbad, CA, USA) and stored at $-80^{\circ} \mathrm{C}$ for several months [16].
At 24 hours before examination or cell seeding, the fresh and cryopreserved AHAMs were rehydrated with sterile PBS, cut into approximately $1.5 \mathrm{~cm} \times 1.5 \mathrm{~cm}$ pieces, which were placed into 24-well cell culture plates with the basement membrane face up, and cultured with MEM-NEAA (Gibco) medium for at least 12 hours.

\section{Cell culture and cell seeding}

hASCs were cultured and differentiated to hepatocytes as described previously [21]. Once the hASC-HLCs were differentiated, these cells were harvested with $0.05 \%$ trypsin-0.02 \% EDTA solution and resuspended in hepatic differentiation medium at a density of $3 \times 10^{5} / \mathrm{ml}$. The primary human hepatocytes (ScienCell Research Laboratories, Carlsbad, CA, USA) were cultured on collagen type I-coated six-well plates $\left(3 \times 10^{5}\right.$ cells/well $)$ with hepatocyte medium (ScienCell Research Laboratories).

The rehydrated cryopreserved AHAM pieces were divided into two groups. In the two-dimensional (2D) group, the AHAM pieces were spread on culture plates and then air-dried for 2 hours before seeding the cells; in the threedimensional (3D) group, the AHAM pieces were spread on culture plates and maintained in MEM-NEAA medium without air-drying before cell seeding. hASC-HLCs were seeded on 2D-AHAM and 3D-AHAM at a density of $1 \times$ $10^{5} / \mathrm{cm}^{2}$, with hASC-HLCs plated on collagen type Icoated 24-well cell culture plates as the control.

\section{Real-time RT-PCR}

Real-time RT-PCR was performed as described previously $[21,22]$. Total cellular RNA was extracted from $3 \times 10^{5}$ cells with the RNeasy Mini Kit (QIAGEN, Hilden, Germany) according to the manufacturer's instructions. For PCR analysis, $1 \mu \mathrm{g}$ RNA was reversetranscribed to cDNA using Superscript III reverse transcriptase and random hexamer primers (Invitrogen, Carlsbad, CA, USA). Real-time PCR analysis was performed on an ABI Prism 7300 Sequence Detection System using the SYBR Green PCR Master Mix (Applied Biosystems, Foster City, CA, USA). The reaction consisted of $10 \mu \mathrm{l}$ SYBR Green PCR Master Mix, $1 \mu \mathrm{l}$ of a $5 \mu \mathrm{M}$ mix of forward and reverse primers, $8 \mu \mathrm{l}$ water, and $1 \mu \mathrm{l}$ template cDNA in a total volume of $20 \mu \mathrm{l}$. Cycling was performed using the default conditions of the ABI 7300 SDS Software 1.3.1 (Applied Biosystems). The relative expression of each gene was normalized against $18 \mathrm{~S}$ rRNA. Data are presented as the mean \pm standard deviation (SD). The primers used are presented in Additional file 1.

\section{Histochemistry and immunofluorescence}

The cells or tissue section were fixed with $4 \%$ paraformaldehyde for 20 minutes at room temperature, followed by permeabilization with $0.3 \%$ Triton X-100 in 
PBS for 5 minutes. The cells were rinsed and blocked with $20 \%$ goat serum (ZSGB-BIO, Beijing, China) or $1 \%$ gelatin (Sigma-Aldrich) for 60 minutes at room temperature. The cells were then incubated with the following antibodies against human antigens rabbit anti-albumin (ALB) at 1:200 (Sigma-Aldrich), rabbit anti-Cytochrome P450 (CYP) 2B6 at 1:50 (Santa Cruz Biotechnology, Dallas, TX, USA), rabbit anti-collagen I at 1:100, rabbit anti-fibronectin at 1:200, mouse anti-collagen IV at 1:100, mouse anti-laminin at 1:200 (ZSGB-BIO), mouse anti-multidrug resistancerelated protein 2 (MRP2) at 1:100 (Santa Cruz), mouse anti-hepatocyte nuclear factor (HNF) $4 \alpha$ at 1:50 (Santa Cruz), and mouse anti-human nuclei at 1:1000 (Millipore, Darmstadt, Germany) or rat monoclonal antibody against mouse CD31 at 1:50 (Santa Cruz) at $4{ }^{\circ} \mathrm{C}$ overnight. A Vector ${ }^{\ominus}$ M.O.M. ${ }^{\mathrm{m}}$ immunodetection kit (Vector Laboratories, Inc., Burlingame, CA, USA) was used according to the manufacturer's protocol to detect the mouse primary monoclonal antibodies for HNF4 $\alpha$ and human nuclei on mouse tissues after hASC-HLC-3D-AHAM transplantation. Following three 5-minute washes in PBS with gentle agitation, an Alexa Fluor-conjugated secondary antibody (1:500; Invitrogen) was added, and the samples were incubated for 1 hour at $37{ }^{\circ} \mathrm{C}$. The nuclei were counterstained with 4',6-diamidino-2-phenylindole (DAPI; Sigma-Aldrich). The stained cells or tissue sections were examined under a Leica TCS SP8 confocal microscope (Leica, Wetzlar, Germany). For detecting the expression of CD31, the tissue sections were examined with the PV-6004 Polink-1 HRP DAB detection system and ZLI-9017 DAB Kit (ZSGB-BIO). The tissue sections were examined under an Axio Imager A2 microscope (Zeiss, Oberkochen, Germany).

\section{Scanning electron microscopy}

For scanning electron microscopy (SEM) analysis, the samples were fixed in $3 \%$ glutaraldehyde in $0.1 \mathrm{M}$ phosphate buffer, $\mathrm{pH} 7.2$, for 120 minutes at $4{ }^{\circ} \mathrm{C}$, postfixed in $1 \%$ osmium tetroxide for 60 minutes at room temperature, and dehydrated in $50 \%, 70 \%, 80 \%, 90 \%$, and $100 \%$ ethanol for 10 minutes, respectively. The samples were then air dried, mounted, sputter coated with gold, and examined using a Hitachi S-4800 scanning electron microscope (Hitachi, Tokyo, Japan).

\section{Transmission electron microscopy}

For the ultra-structural analysis, the differentiated cells were fixed in $2.5 \%$ glutaraldehyde in $0.1 \mathrm{M}$ phosphate

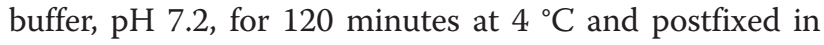
$1 \%$ osmium tetroxide in $0.1 \mathrm{M}$ phosphate buffer. The samples were embedded using the Spurr embedding kit, and sections were examined using a JEM-2100 electron microscope (JEOL, Tokyo, Japan).

\section{ALB analysis}

Medium was harvested following 24 hours of culture for the different cell populations. The ALB content of the culture supernatants was quantified using a commercially available enzyme-linked immunosorbent assay (ELISA) kit (Alpha Diagnostic Intl, San Antonio, TX, USA) according to the manufacturer's protocol.

\section{CYP activity assay}

Ethoxyresorufin-O-deethylase (EROD), methoxyresorufin$\mathrm{O}$-deethylase (MROD), and pentoxyresorufin- $\mathrm{O}$-deethylase (PROD) assays were performed to determine the activities of CYP1A1, CYP1A2, and CYP2B as described previously [21]. The differentiated cells were treated with $1 \mu \mathrm{M} 7$ ethoxyresorufin (Fanbo biochemical, Beijing, China), methoxyresorufin (Fanbo biochemical), and pentoxyresorufin (Sigma-Aldrich) for 24 hours, respectively. The fluorescent products of CYP450 substrates leaked from cells were determined at a wavelength of $585 \mathrm{nM}$ in the absorption under Infnite 200 PRO (Tecan, Männedorf, Switzerland). Quantitative studies employed the high-purity reference standard of resorufin (Fanbo biochemical) for assay standardization.

\section{Bile canaliculus analysis}

The cells were incubated with $10 \mu \mathrm{M}$ 5(6)-carboxy-2,7dichlorofluorescein diacetate (CDFDA; Sigma-Aldrich) at $37{ }^{\circ} \mathrm{C}$ for 10 minutes to allow its internalization and subsequent translocation into the bile canaliculus (BC) lumen by MRP2 to determine the $\mathrm{BC}$ function. After extensive washes, the capacity of the $\mathrm{BC}$ to contain the fluorescent CDF was analyzed under a Leica microscope (Leica) as described previously [21].

\section{Measurement of the plasma amino transferase levels}

The plasma alanine amino transferase (ALT) and aspartate aminotransferase (AST) levels were measured using a Mindray BS-200 analyzer (Mindray, Shenzhen, China).

\section{Acute liver failure induction in mice and hepatic tissue transplantation}

Athymic nude BALB/c male mice aged 6-8 weeks received care according to the Capital Medical University guidelines. All protocols were approved by the Committee for Animal Care. The mice were injected with a single intraperitoneal dose of $\mathrm{CCl} 4$ solution in olive oil (5.0 $\mathrm{ml} / \mathrm{kg}$ body weight as $1 \%$, vol/vol; Sigma-Aldrich) to induce acute liver injury [4]. Vehicle (olive oil)injected mice $(n=3)$ were used as controls.

At 24 hours after CCl4 treatment, hASC-HLCs that were cultured on 3D-AHAM for 3 days and that contained $1 \times 10^{5}$ cells were implanted at the edge of the superior right lobe of the liver with a $0.5 \mathrm{~cm}$ long incision under isoflurane anesthesia. 3D-AHAM pieces without cells were used as controls. The mice were sacrificed at 
days $1,3,7,14$, and 56 post implantation. In each group, there are three to six mice for transplantation. The CCl4 treatment was readministered at the end of another week for the 2-week group, and at the end of every week for the 8-week group. Serum ALT and AST levels were determined at the end of the procedure. Histological analysis of liver tissues was conducted by serial tissue sectioning and staining with hematoxylin \& eosin (H\&E), and the area of injury was assessed using ImageJ software (National Institutes of Health, Bethesda, MD, USA). Human nuclei, ALB, and HNF4 $\alpha$, CYP2B6, and mousespecific CD31 expression were examined at different points by immunofluorescence.

\section{Drug metabolism activity assay}

The drug metabolism activity was analyzed as described previously [23]. Ketoprofen (15 mg/kg; Sigma-Aldrich) was administrated intravenously to the mice post transplantation of the hASC-HLC-3D-AHAM graft or 3DAHAM in the injured liver. Urine was collected 2 hours after administration. Then $100 \mu \mathrm{l}$ urine was mixed with $100 \mu \mathrm{l}$ of $0.5 \mathrm{M}$ acetate buffer (pH 5.0), and $10 \mu \mathrm{l}$ of $1 \mathrm{~N}$ $\mathrm{KOH}$ was added to urine samples, incubated at $80{ }^{\circ} \mathrm{C}$ for 3 hours, neutralized by $10 \mu \mathrm{l}$ of $1 \mathrm{~N} \mathrm{HCl}$, and then centrifuged (15,000 rpm, $4{ }^{\circ} \mathrm{C}, 5$ minutes). The supernatant was subjected to mass spectrometry (Quattro micro API; Waters, Milford, MA, USA). The ionspray voltage was $4500 \mathrm{~V}$ and the analyzed $m / z$ transition (Q1/Q3) for ketoprofen, 1-hydroxyketoprofen, and glucuronide-conjugated ketoprofen was $253.06,269.35$, and 429.34, respectively.

\section{Statistical analysis}

At least three independent determinations of each parameter were compared among the treatment groups by one-way analysis of variance using the statistical software SPSS 11.5 (IBM Corporation, Armonk, NY, USA). Differences were considered significant if $p<0.05$. All data are presented as the mean \pm SD.

\section{Results}

\section{AHAM retained the major components of the AM matrix}

Fresh and treated HAM pieces were examined to establish whether the treatment successfully removed cellular components and to determine the decellularization process. The morphology of the AM surface under phase-contrast microscopy showed that no cells were visible in the treated (Figure S1B in Additional file 2) and cryopreserved (Figure S1C in Additional file 2) HAM pieces compared with the fresh HAM pieces (Figure S1A in Additional file 2). H\&E staining confirmed that the decellularization process was successful (Figure S1E, F in Additional file 2), compared with the fresh HAM pieces (Figure S1D in Additional file 2). SEM analysis demonstrated that the histoarchitecture of the basement membrane was maintained and that no obvious disruption was present following decellularization and cryopreservation in AHAM (Figure S1H, I in Additional file 2), while a single layer of amnion epithelial cells were visible in the fresh HAM (Figure S1G in Additional file 2). Transmission electron microscopy (TEM) analysis demonstrated that a meshwork of collagenous fibrils and stroma were also preserved in AHAM (Figure S1J in Additional file 2).

The HAM pieces were then examined for the presence of major components of the ECM, including collagen type I, collagen type IV, fibronectin, and laminin, before and after decellularization and cryopreservation to determine whether the basement membrane proteins were retained following decellularization. Immunohistochemical analysis showed that these four types of components were all labeled by monoclonal antibodies (Additional file 3). Collagen type I and fibronectin staining were observed in the basement membrane and in the compact layer of the AHAM, and the distribution of collagen type IV and laminin was primarily in the surface of the basement membrane and appeared to be intact in a linear pattern. Therefore, we confirmed that the AHAM retained the natural architecture and components of the AM matrix after decellularization with trypsin-EDTA and cryopreservation with glycerol.

\section{AHAM promotes the functional maturation of the hASC-HLCs}

The hASC-HLCs were seeded on collagen type I-coated cell culture plates and on 2D-AHAM. The morphology of the hepatocytes was then observed using phasecontrast microscopy at different time points to assess the biocompatibility of the AHAM. Within 2 hours after seeding, most of the cells cultured on collagen type I had adhered to the substrate and exhibited irregular shapes; however, the cells cultured on 2D-AHAM remained round. The cells cultured on 2D-AHAM began to adhere at approximately 6 hours after seeding and completely adhered to the AM matrix by 12 hours after seeding. By 72 hours of culture, the cells on collagen type I exhibited typical hepatocyte morphology with a polygonal shape; however, the cells on 2D-AHAM aggregated into clusters containing between 2 and 10 round cells (Additional file 4). Using SEM, the cells cultured on collagen type I appeared markedly flattened, with sharp edges and stiff protrusions (Fig. 1a); however, the morphology of the cells cultured on 2D-AHAM was clearly changed, with a smaller size, spheroidal shape, and abundant villi on the cell surface (Fig. 1b).

Immunofluorescence staining data verified that the cells on 2D-AHAM had significant staining for MRP2 (Fig. 1d), an apical membrane marker of hepatocytes, compared with the cells on collagen type I-coated plates 

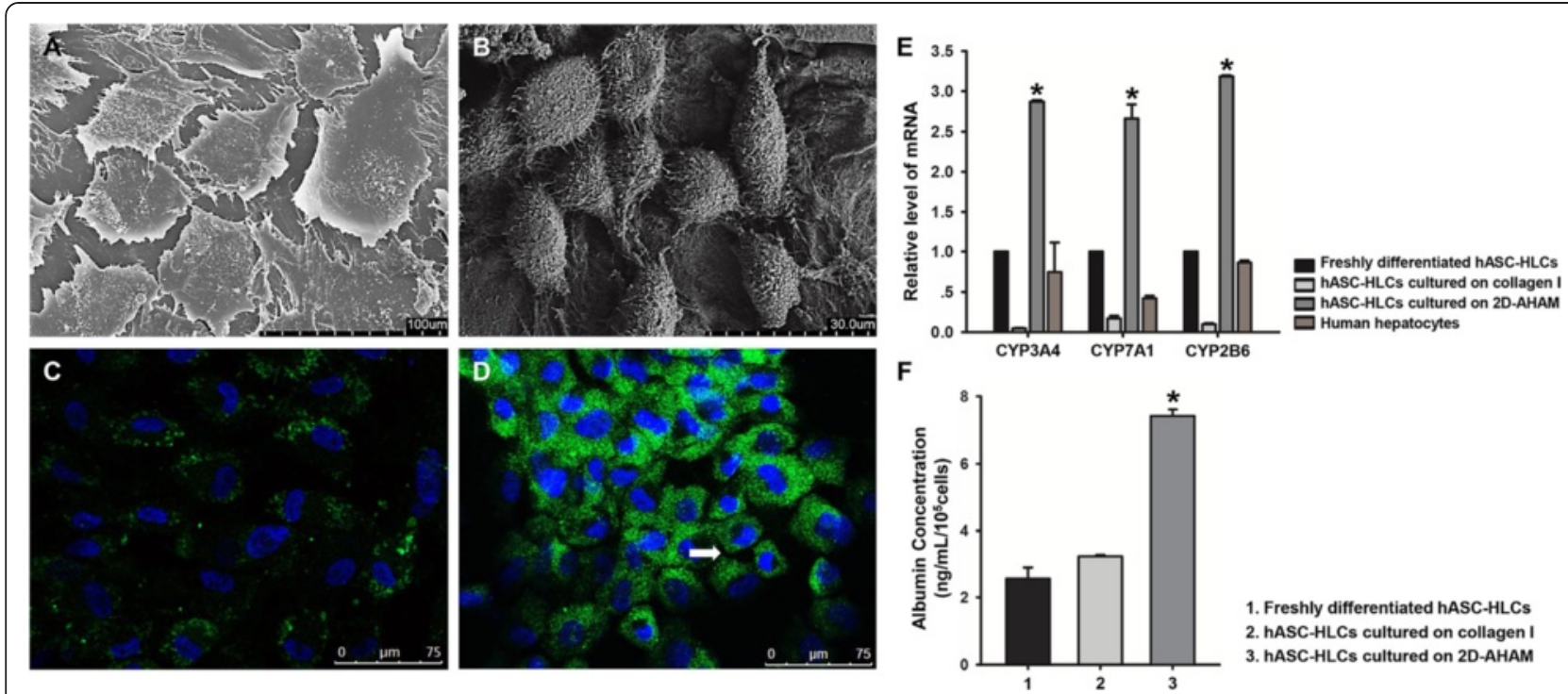

Fig. 1 Properties of hASC-HLCs cultured on collagen type I-coated glass slides and on 2D-AHAM. SEM shows the morphology of hASC-HLCS cultured on collagen type I-coated glass slides (a) and on 2D-AHAM (b) for 72 hours in vitro. Immunofluorescence staining of human MRP2 of hASC-HLCs cultured on collagen type I-coated glass slides (c) and on 2D-AHAM (d). Arrow shows the location of the BC. e Real-time RT-PCR was used to analyze the expression of hepatocyte function-specific genes of hASC-HLCs plated on different substrates. The freshly differentiated hASC-HLCs (indicate that differentiated cells do not trypsinize and reseed after the end of the differentiated program of 21 days) and human hepatocytes were used as controls. The relative expression of each gene was normalized to 185 rRNA. *Statistically significant compared with the hASC-HLCs cultured on collagen type I $(p<0.05)$. $\mathbf{f}$ Levels of ALB secreted by the hASC-HLCs cultured on different substrates as analyzed by ELISA. CYP cytochrome, 2D-AHAM cryopreserved and dried acellular human amniotic membrane, hASC human adipose stem cell, HLC hepatocyte-like cell

(Fig. 1c). To evaluate the functional activity of drug transporters, the cells were cultured with CDFDA, a compound which is metabolized into a fluorescent marker, and transported by polarized cells via MRP2 into BC. The results showed that hASC-HLCs also formed a functional BC structure on the AHAM (Additional file 5).

Real-time RT-PCR analyses showed that the mRNA levels of hepatic metabolism functional markers, including CYP3A4, CYP7A1, and CYP2B6, in the cells cultured on 2D-AHAM were significantly higher than the freshly differentiated cells (in situ), cells on collagen type I, and primary human hepatocytes (Fig. 1e).

The level of ALB secreted by hASC-HLCs cultured on 2D-AHAM was also significantly higher than that produced by the freshly differentiated cells and the cells on collagen type I at 72 hours of culture (Fig. 1f). These results were consistent with the morphological observations, indicating that the AHAM has good biocompatibility with hepatocytes and promotes the functional maturation of hASC-HLCs.

\section{hASC-HLCs reorganized into a hepatic tissue-like structure on 3D-AHAM}

hASC-HLCs were seeded on 3D-AHAM to assess the behaviors and functions of cells on natural AHAM. The cells aggregated into clusters on the AHAM at 24 hours of culture (Fig. 2a), and the cells on the smooth AHAM formed a 3D tissue-like structure by 72 hours of culture (Fig. 2b). Although the cells on 2D-AHAM also aggregated into small clusters after 72 hours of culture (Figure S3F in Additional file 4), they seldom organized into a 3D structure and the AHAM seemed to remain smooth (Fig. 2b insert). Routine histology, immunohistochemical staining, and TEM were performed to examine this 3D tissue-like structure. Upon histological analysis, the hepatocytes appeared well integrated into the AHAM and formed a net-like structure in the center of the tissue (Fig. 2c, d). Additionally, the majority of the cells were ALB-positive (Fig. 2e). The negative control using isotype $\operatorname{IgG}$ for immunofluorescence is shown in Fig. 2e (insert). TEM analysis confirmed that the hepatocytes in cultured hepatic tissues contained abundant mitochondria and that tight junctions and $\mathrm{BC}$ architecture formed between the adjacent hepatocytes (Fig. 2f).

Various hepatocyte characteristics were determined to investigate whether a 3D hepatic tissue-like culture was able to maintain the function of hepatocytes. The expression level of $P C K 2$ gene in the cells cultured on 3DAHAM was higher than those of the cells cultured on 2D-AHAM, but lower than the freshly differentiated cells and primary human hepatocytes. The gene expression levels of CPS1 and APOC1 in the cells cultured on 3D-AHAM were significantly higher than those of the cells cultured on 2D-AHAM and the freshly 

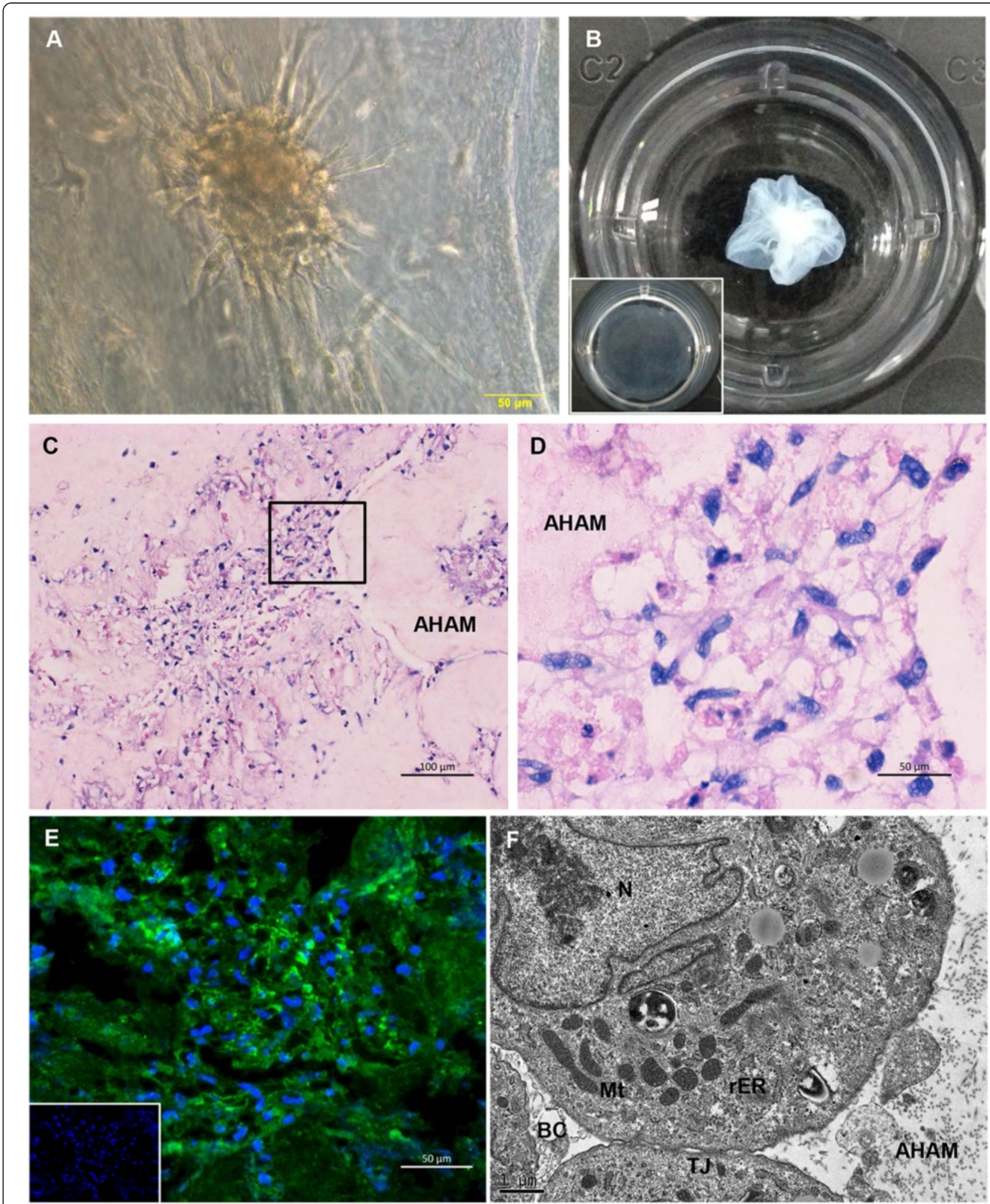

Fig. 2 Properties of hASC-HLCs cultured on 3D-AHAM in vitro. Macroscopic appearance of the hASC-HLC-3D-AHAM cultured on day 1 (a) and day 3 (b) in vitro. Macroscopic appearance of the hASC-HLC-2D-AHAM cultured on day 3 in vitro (b) (insert). H\&E staining showed that the hASC-HLCs attached to the AHAM intensely and formed a 3D nest-like structure (c, d) on day 3. Scale bars: $100 \mu \mathrm{m}$ (c), $50 \mu \mathrm{m}$ (d). e Immunofluorescence staining of ALB of hASC-HLC-3D-AHAM on day 3. The negative control using isotype lgG for immunofluorescence (e) (insert). Scale bar: $50 \mu \mathrm{m}$. f TEM analysis of the ultrastructure of hASC-HLC-3D-AHAM on day 3. Scale bar: $1 \mu \mathrm{m}$. AHAM acellular human amniotic membrane, BC bile canaliculus, Mt mitochondria, $N$ nucleus, $r E R$ rough endoplasmic reticulum, $T J$ tight junction 
differentiated cells, but lower than primary human hepatocytes. Interestingly, the gene expression levels of CYP1A2, CYP2E1,CYP2B6, and CYP3A4 in the cells cultured on 3D-AHAM were significantly higher than those of the freshly differentiated cells, the cells cultured on 2D-AHAM, and primary human hepatocytes. The gene expression levels of CYP7A1 in the cells cultured on 3D-AHAM were significantly higher than those of the freshly differentiated cells and primary human hepatocytes, but there was no difference between the cells cultured on 2D-AHAM and 3D AHAM (Fig. 3).

ALB secretion, ammonia detoxification, and CYP450 activity were detected at day 3 of culture to evaluate the functional activity of hASC-HLCs on 2D-AHAM and 3D-AHAM. The ALB level ( $11 \mathrm{ng} / 10^{5}$ cells/day) secreted by hASC-HLCs cultured on 3D-AHAM was higher than the level secreted by the freshly differentiated cells, and the cells on 2D-AHAM at 72 hours of culture (Fig. 4a).
Similarly, the level of urea production in hASC-HLCs cultured on 3D-AHAM was also higher than that of the freshly differentiated cells and the cells on 2D-AHAM (Fig. 4b). The results presented in Fig. 4c indicate that the activities of CYP1A1 and CYP2B in hASC-HLCs cultured on 3D-AHAM were significantly higher compared with those in hASC-HLCs cultured on 2D-AHAM and the freshly differentiated cells. The activities of CYP1A2 in hASC-HLCs cultured on 3D-AHAM were higher than the freshly differentiated cells, but there was no difference between the cells cultured on 3D-AHAM and 2DAHAM (Fig. 4c).

Taken together, these results suggested that the function of hASC-HLCs cultured on 3D-AHAM was relatively higher than that of the hASC-HLCs cultured on the 2D-AHAM. Moreover, the 3D-AHAM culture condition is likely to be more similar to the in vivo condition than the monolayer culture condition.

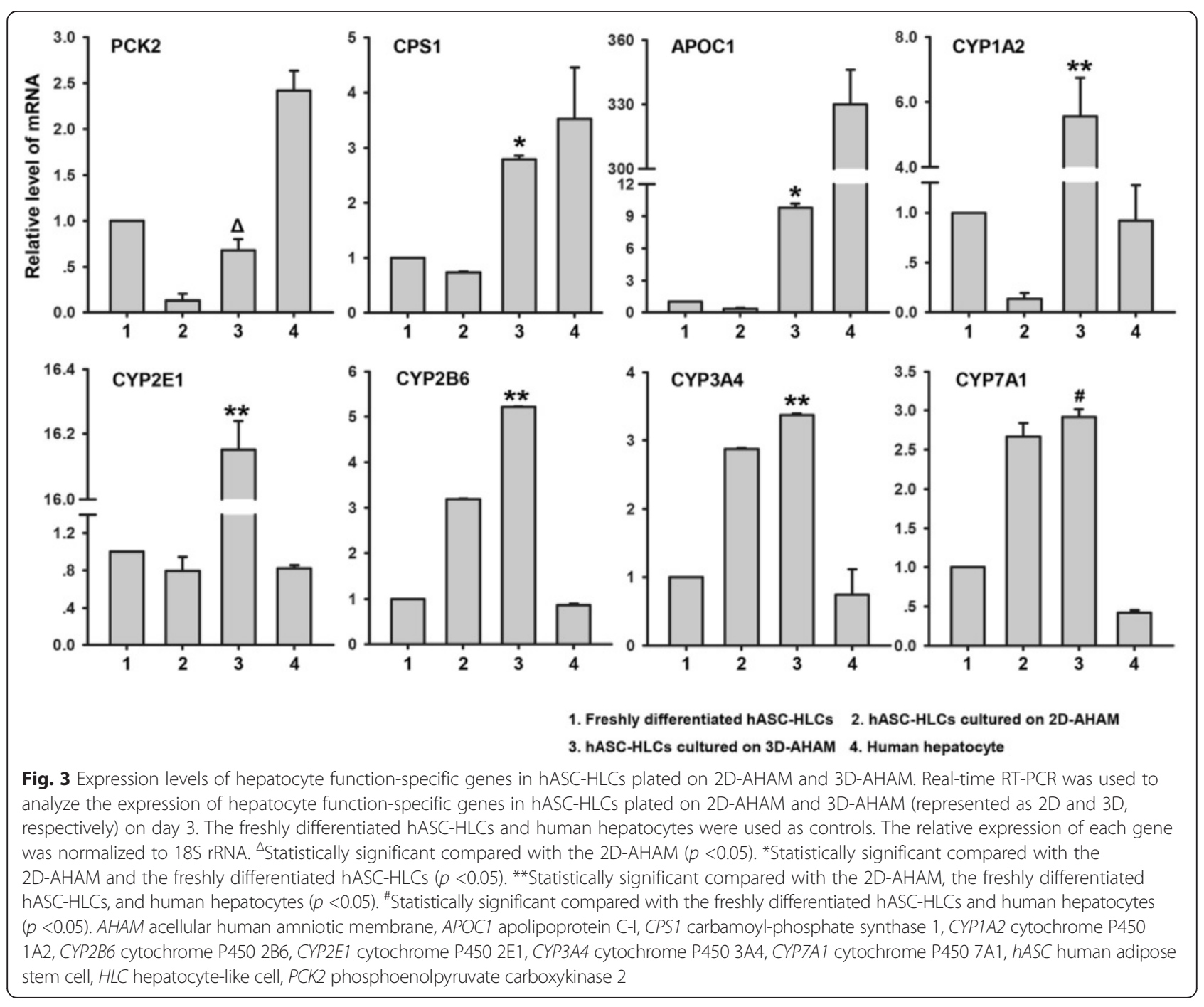



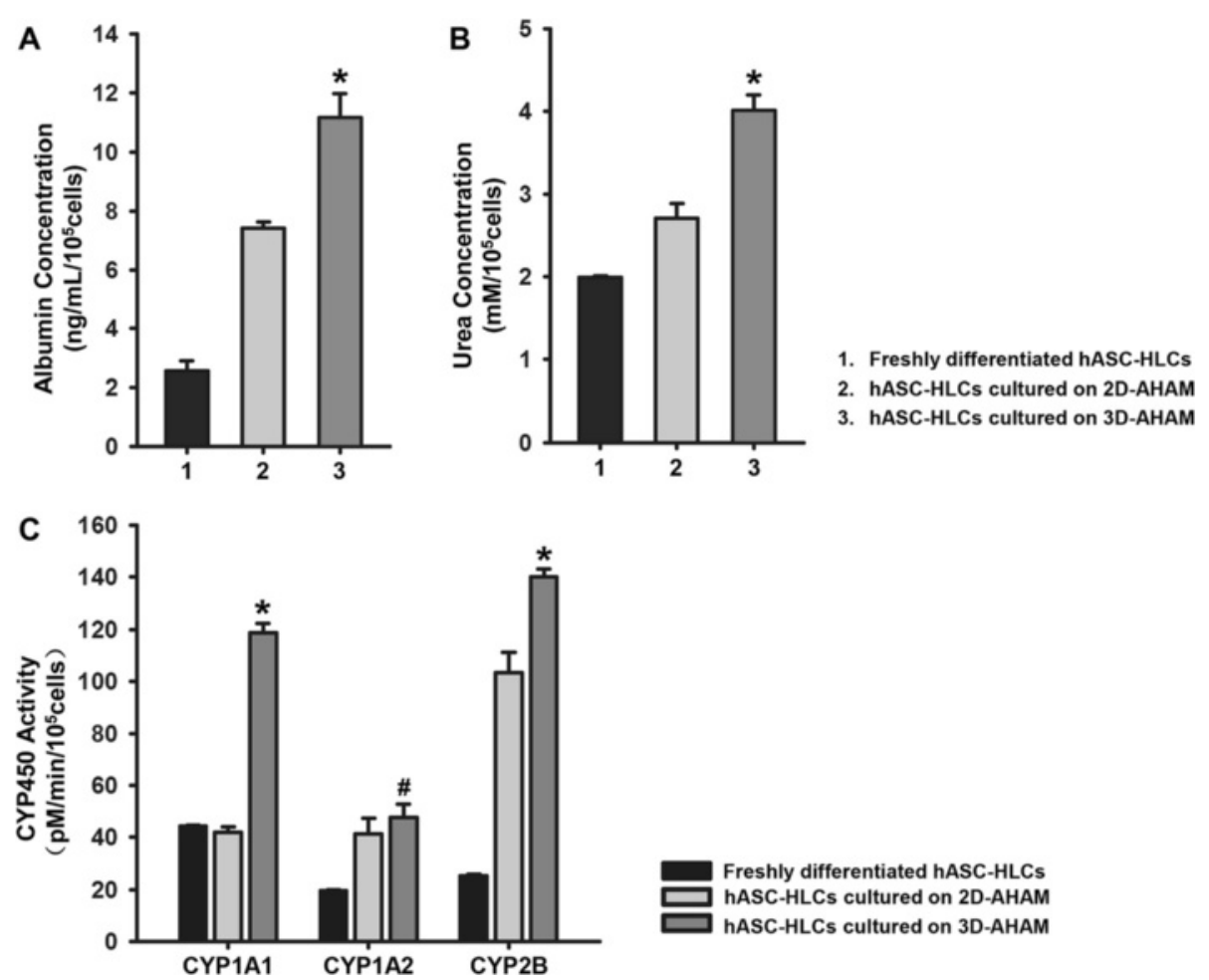

Freshly differentiated hASC-HLCs hASC-HLCs cultured on 2D-AHAM hASC-HLCs cultured on 3D-AHAM

Fig. 4 Functional properties of hASC-HLCs on 3D-AHAM in vitro. a ALB secretion of hASC-HLCs cultured on 2D-AHAM and 3D-AHAM, and freshly differentiated hASC-HLCS (represented as 1, 2, and 3, respectively) analyzed by ELISA. b Ammonia metabolism, as reflected by urea synthesis, was determined in the hASC-HLCs cultured on 2D-AHAM and 3D-AHAM, and freshly differentiated hASC-HLCs (represented as 1, 2, and 3, respectively). c EROD, MROD, and PROD assays for the activities of CYP1A1, CYP1A2, and CYP2B in hASC-HLCs cultured on 2D-AHAM and 3D-AHAM, and freshly differentiated hASC-HLCs. *Statistically significant compared with the hASC-HLCs cultured on 2D-AHAM and freshly differentiated hASC-HLCs $(p<0.05)$. "Statistically significant compared with the freshly differentiated hASC-HLCs $(p<0.05)$. AHAM acellular human amniotic membrane, CYP cytochrome, hASC human adipose stem cell, HLC hepatocyte-like cell

\section{hASC-HLC-3D-AHAM constructs implant into the livers of immunodeficient mice}

The hASC-HLC-3D-AHAM constructs were implanted into an immunodeficient mouse model with $\mathrm{CCl} 4$ treatment to test whether the hASC-HLC-3D-AHAM constructs can engraft after transplantation. The acute liver injury induced by a single-dose injection of CCl4 was evidenced by an increase in the plasma ALT level to $>2500$ $\mathrm{U} / \mathrm{l}$ (normal range, $<50 \mathrm{U} / \mathrm{l}$ ), by an increase in the plasma AST level to $>2200 \mathrm{U} / \mathrm{l}$ (normal range, $<80 \mathrm{U} / \mathrm{l}$ ), and by larger necrotic areas around the central venous as determined by histochemistry analysis 24 hours after $\mathrm{CCl} 4$ treatment (Additional file 6).

Acute liver injury was validated by analyzing the massive necrosis during histological examination and the serum ALT levels in the injured mice with the hASC-HLC-3DAHAM construct or with AHAM only to evaluate the beneficial effect of the hASC-HLC-3D-AHAM construct on the recovery of the $\mathrm{CCl} 4$-injured liver after implantation. H\&E staining (Fig. 5a, b) showed that the areas of inflammation, sinusoid congestion, and hemorrhage decreased and that the serum ALT level decreased significantly in mice implanted with hASC-HLC-3DAHAM constructs compared with the mice implanted with AHAM only (Fig. 5c, d). Interestingly, the transplanted AHAM only attached the middle of the liver lobes and did not integrate with the liver tissue (Fig. 5a). In contrast, approximately $33 \%$ of the mice $(n=6)$ displayed integrated hASC-HLC-3D-AHAM transplants (Fig. 5b). The results suggested that the hASC-HLC-3D-AHAM engraftment might alleviate the liver injury induced by $\mathrm{CCl} 4$.

Histological and immunohistochemical analyses were performed at days 3,7 , and 14 post transplantation to detect the engraftment of the transplanted hASC-HLC3D-AHAM constructs. The results showed that the hASC-HLC-3D-AHAM constructs had engrafted into the livers of the mice at day 3 post transplantation (Fig. 6a). H\&E staining showed that the tissue-like constructs were restricted to the cut edge of each liver and that the cells in this construct formed large net-like structures (Fig. 6b, c). Immunohistochemical analysis confirmed that the cells in the construct stained positive for human nuclei, ALB, and HNF4 $\alpha$ (Fig. 6d-f). Data in 

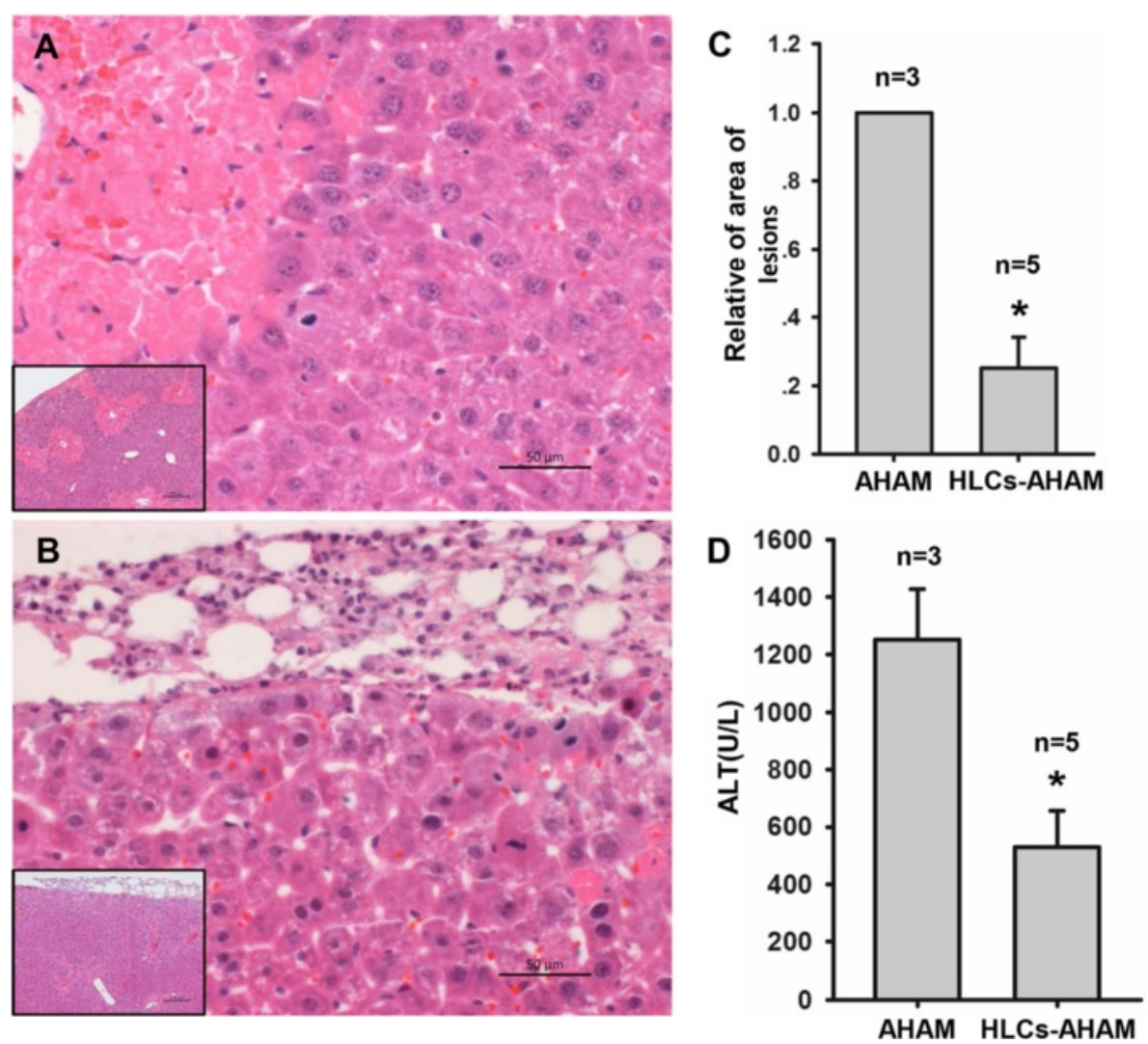

Fig. 5 Semiquantitative evaluation of lesions in liver tissue sections following transplantation. H\&E staining analysis of the lesions in the liver tissue at 24 hours after the transplantation of AHAM $(\mathbf{a})(n=3)$ or hASC-HLC-3D-AHAM grafts $(\mathbf{b})(n=5)$ in a CCl4-induced liver injury immunodeficient mouse model. c Percentage of lesion area was evaluated using Image J software. $\mathbf{d}$ Plasma levels of ALT were detected. *Statistically significant compared with the AHAM graft $(p<0.05)$. AHAM acellular human amniotic membrane, ALT alanine amino transferase, HLC hepatocyte-like cell

Additional files 7 and 8 reveal that the hASC-HLC-3DAHAM constructs remained integrated into the liver at days 7 and 14 post transplantation, with large clusters of hepatocytes arranged into cord-like structures, and the cells in the construct were also positive for human nuclei, ALB, and HNF4 $\alpha$. The results suggested that the AHAM engraftment of hASC-HLCs in vivo was highly efficient.

The fate of transplanted hASC-HLC-3D-AHAM constructs in mice was considered in the long term. $H \& E$ staining showed that the tissue-like constructs were completely integrated to the liver at day 56 post transplantation, and the morphology of cells in grafts near the edge of the host changed to cubic (Fig. 7a). There was no evidence of abnormal proliferation or regression in the graft. Immunohistochemical analysis confirmed that the cells in the construct stained positive for human nuclei (Fig. 7b), and for hepatic functional marker ALB (Fig. 7c), HNF4 $\alpha$ (Fig. 7d), and CYP2B6 (Fig. 7e).

The functional maturation of hASC-HLCs in transplants was evaluated using the drug metabolism activity assay. Mice transplanted with the hASC-HLC-3DAHAM constructs or 3D-AHAM were challenged with ketoprofen, which are known to be metabolized differently by mice and humans. After the drug exposure, formation of metabolites in urine samples from mouse or human specific was collected and determined. Results showed that the 1-hydroxyketoprofen was detected in mice transplanted with hASC-HLC-3D-AHAM constructs or 3D-AHAM. However, the glucuronideconjugated ketoprofen was only detected in the urea sample of mice transplanted with hASC-HLCs at day 56 post transplantation (Additional file 9). Interestingly, H\&E staining showed that the vessel-like structures presented in the transplanted hASC-HLC-3D-AHAM graft (Fig. 7a). Immunohistochemical analysis confirmed that cells composed the vessel-like structures were positive for mouse CD31 antibody (Fig. 7f). These results demonstrate that hASC-HLCs maintained the property of functional maturation, and the potential functional vessels in grafts were connecting with host vessels when hASC-HLC-3D-AHAM grafts were transplanted in the long term. 

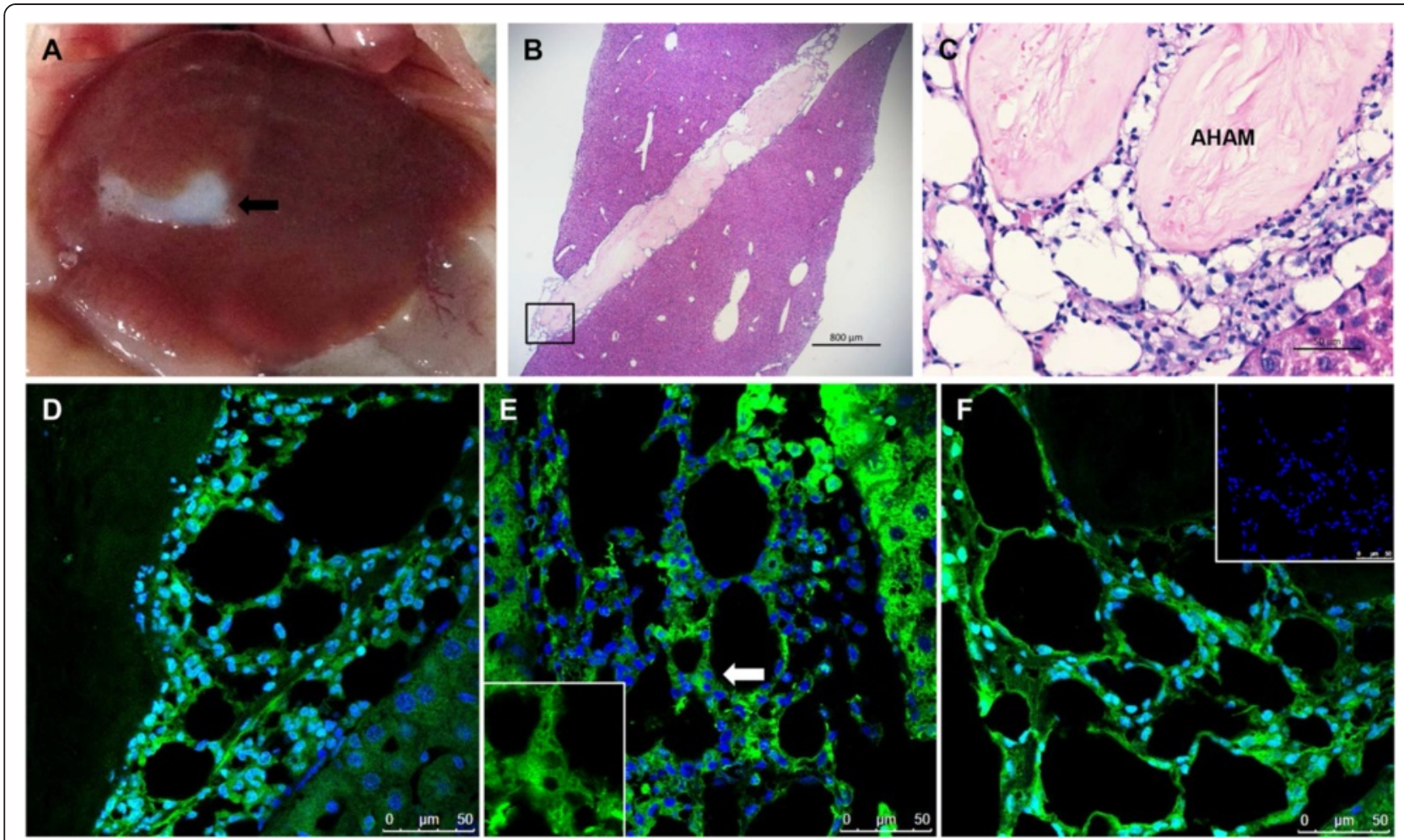

Fig. 6 Implantation of the hASC-HLC-3D-AHAM graft into the CCI4-injured liver. a Gross appearance of the hASC-HLC-3D-AHAM graft in the injured liver at 3 days post implantation. Arrow shows the location of the graft. $\mathbf{b}, \mathbf{c} H \& E$ staining analysis of the graft integrated with the liver tissue. Scale bars: $800 \mu \mathrm{m}$ b, $50 \mu \mathrm{m} \mathbf{c}$. Immunofluorescence staining of human nuclei (d), ALB (e), and HNF4a (f) in the hASC-HLC-3D-AHAM graft in the CCl4-injured liver. Scale bar: $50 \mu \mathrm{m}$. Negative control using isotype lgG for immunofluorescence (f) (insert). AHAM acellular human amniotic membrane

Our study has demonstrated that hASC-HLC-3DAHAM transplantation offers an alternative approach to the generation of a 3D, transplantable hepatic graft. These results offered the therapeutic potential using autohepatocytes derived from patient stem cells for treating liver failure.

\section{Discussion}

The use of hASC-derived hepatocytes as regenerative cells in hepatic disease would be advantageous for ethical and safety reasons, and these autologous cells are immunocompatible, exhibit controlled differentiation, and do not undergo post-transplantation rejection [3]. Thus far, the low engraftment of the available hepatocytes has been a major obstacle [24]. Recently, hepatocytes that have been assembled on 3D decellularized matrices to generate liver tissues or patches have become a novel platform for transplantation $[14,25,26]$. In the present study, we provide evidence that the AHAM has good biocompatibility with hepatocytes and promotes the functional maturation of hASC-HLCs. Moreover, the natural AHAM may induce the reorganization of hASC-HLCs into a hepatic tissuelike structure in vitro, which efficiently alleviated the acute liver injury, was integrated with the liver tissue at the implantation site, and induced hepatic plate-like structure in the grafts after transplantation into $\mathrm{CCl} 4$-injured livers of immunodeficient mice.

The AM, which is the innermost layer of the placenta, is composed of an amniotic epithelial cell layer, a basement membrane, and a thick compact layer or avascular stroma. The special structure and biological viability of the amniotic ECM plays a major role in providing mechanical strength to the amniotic epithelial cells [18]. Previous evidence showed that the acellular matrix nature of AM also functions as a primary determinant of attachment, survival, differentiation, and cytoskeletal organization for epithelial cells of other origins [17], such as pneumocytes [27], limbal epithelial cells $[28,29]$, salivary gland cells [30], and dental apical papilla cells [31]. The AHAM may therefore be a reasonable native scaffold for hepatocyte seeding.

Retaining the biochemical composition and architecture of the matrix in intact AM following the decellularization process is a critical step for natural scaffold preparation. First, we demonstrated that the amniotic epithelial cells were removed completely from the AM using $0.25 \%$ trypsin and $0.38 \%$ EDTA without any mechanical scraping [16]. The architecture of the AM 

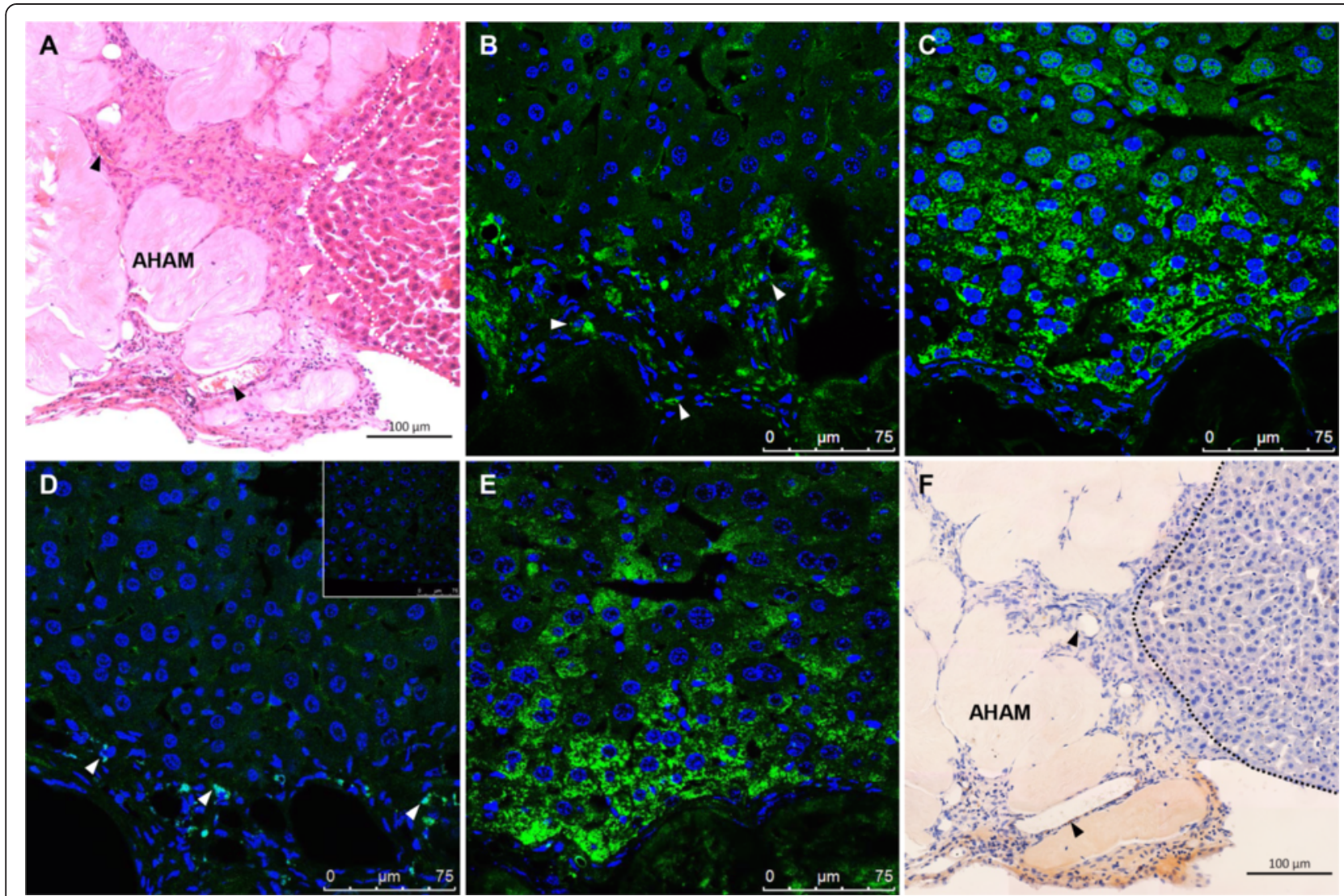

Fig. 7 Long-term properties of the hASC-HLC-3D-AHAM graft in the injured liver following transplantation. a H\&E staining analysis of the graft at 8 weeks post implantation. Black arrows show the location of the vessel-like structure. White arrows show the hASC-HLCs with the cubic morphology. Immunofluorescence staining of human nuclei (b), ALB (c), HNF4a (d), CYP2B6 (e), and mouse CD31 (f) in the hASC-HLC-3D-AHAM graft in the CCl4-injured liver at 8 weeks post implantation. The control of immunofluorescence using anti-human nuclei was analyzed in tissues from nontransplanted mice (d) (insert). Black arrows show the location of the vessel-like structure. Dotted lines show the location of the grafts. Scale bar: $75 \mu \mathrm{m}$ (b), (c), $100 \mu \mathrm{m}(\mathbf{a}),(\mathbf{d})$. AHAM acellular human amniotic membrane

matrix, including a smooth and compact collagenous basement surface and a meshwork of collagenous fibrils and stroma, was preserved upon decellularization and cryopreservation (Additional file 2). Immunostaining analysis suggested that the matrix of collagen type I, collagen type IV, fibronectin, and laminin found in the hepatocyte niche remained relatively intact compared with that of the fresh AM (Additional file 3). Then, we compared the morphological appearance and function of hASC-HLCs when seeded on conventional collagen type I-coated plates and 2D-AHAM configurations. The results showed that the hASC-HLCs cultured on 2DAHAM appeared round or spheroidal, which is the desirable morphology for the functional maintenance of hepatocytes, versus the flattened and spread polygonal cells cultured on collagen type I as shown previously (Fig. 1) [21]. Consistent with these findings, quantitative gene expression analysis illustrated that hASC-HLCs cultured on 2D-AHAM exhibited a significant increase in hepatocyte function-specific gene expression at day 3 than the freshly differentiated cells, the cells cultured on collagen type I, and human hepatocytes (Fig. 1e). An analogous increase in functional properties, including CDFDA metabolism and ALB secretion, was observed in hASC-HLCs cultured on 2D-AHAM (Fig. 1; Additional file 5). These data demonstrated that the matrix components of AHAM have an effect on maintaining the hepatocyte phenotype. The accumulated evidence suggested that both collagen types I and IV, as well as the widely distributed adhesive protein fibronectin, play an important role in modulating the formation of hepatocyte polarity, such as the organization of cytoskeletal proteins and the expression of a wide array of liver-specific functions [32]. The AHAM may also be a favorable substrate for hepatocyte carriers.

Next, we examined whether hASC-HLCs could attach to the natural AM ECM (3D-AHAM), which not only preserved the components of the AM matrix but also retained the specific 3D architecture of the AM matrix. The results showed that hASC-HLCs self-organized into 
microscopically visible 3D cell clusters by an intrinsic organizing capacity after 24 hours of culture on 3D-AHAM. The macroscopically visible clusters continued to form for up to 72 hours of culture (Fig. 2). We visualized the formation of homogeneously distributed hASC-HLCs in this construct as detected by histology, immunostaining, and TEM. Similarly, the levels of ALB secretion, urea synthesis, and CYP450 enzyme activity were elevated in hASC-HLCs grown on 3D-AHAM compared with their counterparts grown on 2D-AHAM and the freshly differentiated cells (Fig. 4). This result suggests that the 3D-AHAM microenvironment or spatial organization may provide more effective signals, such as the surface texture, variation in pore diameter, spatial presentation of the surface proteins, and mechanical strength for the hASC-HLCs $[32,33]$.

The efficiency of the hASC-HLC-3D-AHAM constructs for hepatocyte transplantation was further assessed using a grafting protocol. Our study dramatically demonstrated that the hASC-HLC-3D-AHAM engraftment alleviated the acute liver injury on day 1 and the efficiency was restricted to the desired target sites on day 3 post transplantation as determined by histological examination. The fate of transplanted hASC-HLC-3D-AHAM constructs was further examined 2 months post transplantation. Similarly, the constructs were integrated in the liver of mice as before without multiplying and regressively repopulating. For assessing progressive humanization or the functional maturation of hASC-HLCs in host liver, the ketoprofen metabolism was performed in vivo to validate the functionality of engrafted cells and the functional vessel formation in transplanted hASC-HLC-3D-AHAM constructs. Ketoprofen is a propionic acid-class nonsteroidal anti-inflammatory drug with analgesic and antipyretic effects, which are known to be metabolized differently by mice and humans [23, 34]. Ketoprofen is primarily metabolized by CYP450s in mice to form 1hydroxyketoprofen, while in humans ketoprofen is mainly metabolized by UDP-glucuronosyltransferase to form ketoprofen glucuronide by hydrolysis [35]. We observed that the glucuronide-conjugated ketoprofen was detected in urea in mice transplanted with hASC-HLC-3D-AHAM using mass spectrometry. The histological and immunohistochemical analysis further demonstrated the functional vessel structure formed in the transplanted hASC-HLC-3D-AHAM constructs.

\section{Conclusions}

The present study suggests that the AHAM has the ability to maintain the functional phenotype of the hASCHLCs and is transplantable. This approach sounds rather straightforward; alternatives that would aid in providing surgical potential to manage the bottleneck of donor liver availability for both organ and hepatocyte transplantation are therefore worth discussing. Our findings, combined with the development of tissue engineering technologies, may support adult stem cell-based therapy for future individualized clinical therapy of liver failure and for bioartificial liver establishment.

\section{Additional files}

Additional file 1: is Table $\mathrm{S} 1$ presenting primers for real-time RT-PCR. (DOC 35 kb)

Additional file 2: is Figure $\mathrm{S} 1$ showing characterization of fresh HAM and AHAM. The properties of fresh HAM $(A, D, G)$, fresh AHAM $(B, E, H)$, and cryopreserved AHAM $(C, F, I)$ were determined by phase microscopy (A, B, C), H\&E staining (D, E, F), SEM $(G, H, I)$ and TEM (J). Scale bars: $50 \mu \mathrm{m}$ A-F. (TIFF $7208 \mathrm{~kb}$ )

Additional file 3: is Figure $\mathrm{S} 2$ showing distribution of ECM components in fresh HAM and in cryopreserved AHAM. Immunohistological evaluation of fresh HAM and cryopreserved AHAM before application. Scale bars: $50 \mu \mathrm{m}$. HAM human amniotic membrane, AHAM acellular human amniotic membrane, I collagen type I, IV collagen type IV, FN fibronectin, LN laminin. (TIFF $9626 \mathrm{~kb}$ )

Additional file 4: is Figure $\mathrm{S} 3$ showing morphological properties of hASC-HLCs on different matrices. Morphology of hASC-HLCs cultured on collagen type I-coated 24-well cell culture plates (A, C, E) and on cryopreserved 2D-AHAM (B, D, F) was examined by phase microscopy at 6 hours (A, B), 12 hours (C, D) and 72 hours (E, F) after cell seeding. Scale bars: $50 \mu \mathrm{m}$. (TIFF $9815 \mathrm{~kb}$ )

Additional file 5: is Figure $\mathbf{S 4}$ showing $\mathrm{BC}$ analysis. CDFDA is internalized by hASC-HLCs cultured on collagen type I-coated glass slides (A) and on 2D-AHAM (B), cleaved by intracellular esterases, and excreted into the $B C$ as fluorescent CDF. Arrow shows the location of the fluorescent CDF. Scale bars: $50 \mu \mathrm{m}$. (TIFF $5628 \mathrm{~kb}$ )

Additional file 6: is Figure $\mathrm{S} 5$ showing acute liver injury in $\mathrm{BALB} / \mathrm{C}$ nude mice induced with CCL4. The general appearance of the liver (A) and H\&E staining (B) in BALB/C nude mice that were intraperitoneally injected with olive oil. Gross appearance of the liver (C) and H\&E staining (D) in BALB/C nude mice that were intraperitoneally injected with CCL4. At 24 hours post injection, white spots were found at the surface of the liver (C), and H\&E staining (D) showed more numerous and larger necrotic areas in the liver around the central venous (black arrow). Scale bar: $100 \mu \mathrm{m}$. Plasma levels of ALT (E) and AST (F) were detected in the CCl4-induced acute liver injury group and in the control. *Statistically significant compared to the control group $(p<0.05)$. (TIFF $7839 \mathrm{~kb})$

Additional file 7: is Figure S6 showing properties of the hASC-HLC3D-AHAM graft in the injured liver at day 7 following transplantation. (A) H\&E staining analysis of the graft at day 7 post implantation. Scale bar: $100 \mu \mathrm{m}$. Immunofluorescence staining of human nuclei (B), ALB (C), and HNF4a (D) in the hASC-HLC-3D-AHAM graft in the CCL4-injured liver at 1 week post implantation. $\lg G$ as negative control $(B$, insert). Scale bar: $50 \mu \mathrm{m}$. (TIFF $9840 \mathrm{~kb}$ )

Additional file 8: is Figure S7 showing properties of the hASC-HLC3D-AHAM graft in the injured liver at day 14 following transplantation. (A) H\&E staining analysis of the graft at day 14 post implantation. Immunofluorescence staining of human nuclei (B), ALB (C), and HNF4a (D) in the hASC-HLC-3D-AHAM graft in the CCL4-injured liver at 2 weeks post implantation. IgG as negative control (B, insert). Scale bar: $50 \mu \mathrm{m}$. (TIFF $9846 \mathrm{~kb}$ )

Additional file 9: is Figure $\mathrm{S} 8$ showing drug metabolism activity of the hASC-HLC-3D-AHAM graft in the mouse liver at day 56 post implantation. Ketoprofen (15 mg/kg; Sigma-Aldrich) was administrated intravenously to the mice post transplantation of the hASC-HLC-3DAHAM graft $(A, B, C)$ or 3D-AHAM $(D, E, F)$ in the injured liver. Urine was collected 2 hours after administration. Then $100 \mu$ urine was mixed with $100 \mu \mathrm{l}$ of $0.5 \mathrm{M}$ acetate buffer ( $\mathrm{pH} 5.0)$, and then $10 \mu \mathrm{l}$ of $1 \mathrm{~N} \mathrm{KOH}$ was 
added to urine samples, incubated at $80^{\circ} \mathrm{C}$ for 3 hours, neutralized by $10 \mu \mathrm{l}$ of $1 \mathrm{~N} \mathrm{HCl}$, and then centrifuged $\left(15,000 \mathrm{rpm}, 4^{\circ} \mathrm{C}, 5\right.$ minutes). The supernatant was subjected to mass spectrometry (Quattro micro API; Waters). The ionspray voltage was $-4500 \mathrm{~V}$ and analyzed $\mathrm{m} / \mathrm{z}$ transition (Q1/Q3) for ketoprofen,1-hydroxyketoprofen, glucuronide-conjugated ketoprofen was 253.06, 269.35, and 429.34, respectively. (B) Amplification of part 1, (C) amplification of part 2, (E) amplification of part 3, (F) amplification of part 4. Arrow shows the location of the ketoprofen, 1-hydroxyketoprofen, glucuronide-conjugated ketoprofen, OH-ketoprofen: 1-hydroxyketoprofen; G-ketoprofen: glucuronide-conjugated ketoprofen. (TIFF $9829 \mathrm{~kb})$

\section{Abbreviations}

AHAM: Acellular human amniotic membrane; ALB: Albumin; ALT: Alanine amino transferase; AM: Amniotic membrane; APOC1: Apolipoprotein C-1; AST: Aspartate aminotransferase; BC: Bile canaliculus; CCl4: Carbon tetrachloride; CDFDA: 5(6)-Carboxy-2,7-dichlorofluorescein diacetate; CPS1: Arbamoyl-phosphate synthase 1; CYP: Cytochrome; 2D: Twodimensional; 3D: Three-dimensional; DAPI: 4',6-Diamidino-2-phenylindole; ECM: Extracellular matrix; EDTA: Ethylenediamine tetraacetic acid; ELISA: Enzyme-linked immunosorbent assay; EROD: Ethoxyresorufin-Odeethylase; HAM: Human amniotic membrane; hASC: Human adipose stem cell; H\&E: Hematoxylin \& eosin; HLC: Hepatocyte-like cell; HNF: Hepatocyte nuclear factor; MROD: Methoxyresorufin-O-deethylase; MRP2: Multidrug resistance-related protein 2; PBS: Phosphate-buffered saline; PCK2: Phosphoenolpyruvate carboxykinase 2; PROD: Pentoxyresorufin-Odeethylase; SD: Standard deviation; SEM: Scanning electron microscopy; TEM: Transmission electron microscopy.

\section{Competing interests}

The authors indicate no potential conflicts of interest.

\section{Authors' contributions}

JY was responsible for conception and design, collection and assembly of data, and data analysis and interpretation. WL was responsible for administrative support, and collection and assembly of data. JH was responsible for collection and assembly of data. XG was responsible for collection and assembly of data. XLi was responsible for collection data. XLu was responsible for collection data and histochemistry technical support. XH was responsible for collection and assembly of data. $\mathrm{HZ}$ was responsible for conception and design, data analysis and interpretation, and final approval of the manuscript. All authors read and approved the manuscript.

\section{Acknowledgements}

This research was supported by the National Natural Science Foundation of China (No. 31171310), and the Project of Construction of Innovative Teams and Teacher Career Development for Universities and Colleges under Beijing Municipality (No. IDHT20150502). The authors thank Prof. Liying Li, Qing Cai, and Jun Deng for advice in performing these experiments.

\section{Funding}

This work was supported by the National Natural Science Foundation of China (No. 31171310) and The Project of Construction of Innovative Teams and Teacher Career Development for Universities and Colleges under Beijing Municipality (IDHT20150502).

\section{Author details}

'Department of Cell Biology, Municipal Laboratory for Liver Protection and Regulation of Regeneration, Capital Medical University, No. 10, Xitoutiao, You An Men, Beijing 100069, China. ${ }^{2}$ Fu Xing Hospital, Capital Medical University, No. 20, Fu xing men wai, Beijing 100038, China.

Received: 25 January 2015 Revised: 31 July 2015 Accepted: 19 August 2015 Published online: 05 November 2015

\section{References}

1. Duncan AW, Dorrell C, Grompe M. Stem cells and liver regeneration. Gastroenterology. 2009;137(2):466-81.
2. Dhawan A, Puppi J, Hughes RD, Mitry RR. Human hepatocyte transplantation: current experience and future challenges. Nat Rev Gastroenterol Hepatol. 2010;7(5):288-98.

3. Mizuno $H$, Tobita M, Uysal AC. Concise review: Adipose-derived stem cells as a novel tool for future regenerative medicine. Stem Cells. 2012;30:804-10.

4. Banas A, Teratani T, Yamamoto Y, Tokuhara M, Takeshita F, Quinn G, et al. Adipose tissue-derived mesenchymal stem cells as a source of human hepatocytes. Hepatology. 2007:46(1):219-28.

5. Mooney DJ, Vandenburgh $\mathrm{H}$. Cell delivery mechanisms for tissue repair. Cell Stem Cell. 2008;2:205-13.

6. Nussler A, Konig S, Ott M, Sokal E, Christ B, Thasler W, et al. Present status and perspectives of cell-based therapies for liver diseases. J Hepatol. 2006;45(1):144-59.

7. Hoppo T, Komori J, Manohar R, Stolz DB, Lagasse E. Rescue of lethal hepatic failure by hepatized lymph nodes in mice. Gastroenterology. 2011;140(2):656-66.

8. Chen AA, Thomas DK, Ong LL, Schwartz RE, Golub TR, Bhatia SN. Humanized mice with ectopic artificial liver tissues. Proc Natl Acad Sci U S A. 2011;108(29):11842-7.

9. Navarro-Alvarez N, Soto-Gutierrez A, Chen Y, Caballero-Corbalan J, Hassan W, Kobayashi S, et al. Intramuscular transplantation of engineered hepatic tissue constructs corrects acute and chronic liver failure in mice. J Hepatol. 2010;52(2):211-9.

10. Ohashi K, Waugh JM, Dake MD, Yokoyama T, Kuge H, Nakajima Y, et al. Liver tissue engineering at extrahepatic sites in mice as a potential new therapy for genetic liver diseases. Hepatology. 2005;41(1):132-40.

11. Uygun BE, Yarmush ML. Engineered liver for transplantation. Curr Opin Biotechnol. 2013;24(5):893-9.

12. Uygun BE, Soto-Gutierrez A, Yagi H, Izamis ML, Guzzardi MA, Shulman C, et al. Organ reengineering through development of a transplantable recellularized liver graft using decellularized liver matrix. Nat Med. 2010;16(7):814-20.

13. Vasanthan KS, Subramanian A, Krishnan UM, Sethuraman S. Role of biomaterials, therapeutic molecules and cells for hepatic tissue engineering. Biotechnol Adv. 2012;30(3):742-52.

14. Turner RA, Wauthier E, Lozoya O, McClelland R, Bowsher JE, Barbier C, et al. Successful transplantation of human hepatic stem cells with restricted localization to liver using hyaluronan grafts. Hepatology. 2013;57(2):775-84.

15. Niknejad H, Peirovi $H$, Jorjani M, Ahmadiani A, Ghanavi J, Seifalian AM. Properties of the amniotic membrane for potential use in tissue engineering. Eur Cell Mater. 2008;15:88-99.

16. Riau AK, Beuerman RW, Lim LS, Mehta JS. Preservation, sterilization and de-epithelialization of human amniotic membrane for use in ocular surface reconstruction. Biomaterials. 2010;31(2):216-25.

17. Wilshaw SP, Kearney JN, Fisher J, Ingham E. Production of an acellular amniotic membrane matrix for use in tissue engineering. Tissue Eng Part A. 2006;8:2117-29.

18. Aplin JD, Campbell S, Allen TD. The extracellular matrix of human amniotic epithelium: ultrastructure, composition and deposition. J Cell Sci. 1985;79:119-36.

19. Sant'Anna LB, Cargnoni A, Ressel L, Vanosi G, Parolini O. Amniotic membrane application reduces liver fibrosis in a bile duct ligation rat model. Cell Transplant. 2011;20(3):441-53.

20. Ricci E, Vanosi G, Lindenmair A, Hennerbichler S, Peterbauer-Scherb A Wolbank S, et al. Anti-fibrotic effects of fresh and cryopreserved human amniotic membrane in a rat liver fibrosis model. Cell Tissue Bank. 2013;14(3):475-88

21. Li X, Yuan J, Li W, Liu S, Hua M, Lu X, et al. Direct differentiation of homogeneous human adipose stem cells into functional hepatocytes by mimicking liver embryogenesis. J Cell Physiol. 2014;229(6):801-12.

22. Hua M, Zhang W, Li W, Li X, Liu B, Lu X, et al. Molecular mechanisms regulating the establishment of hepatocyte polarity during human hepatic progenitor cell differentiation into a functional hepatocyte-like phenotype. J Cell Sci. 2012;125(Pt 23):5800-10.

23. Takebe $\mathrm{T}$, Sekine $\mathrm{K}$, Enomura M, Koike H, Kimura M, Ogaeri T, et al. Vascularized and functional human liver from an iPSC-derived organ bud transplant. Nature. 2013:499(7459):481-4.

24. Aurich $H$, Sgodda M, Kaltwasser $P$, Vetter $M$, Weise A, Liehr T, et al. Hepatocyte differentiation of mesenchymal stem cells from human adipose tissue in vitro promotes hepatic integration in vivo. Gut. 2009;58(4):570-81.

25. Baeck C, Streetz K. The recellularized liver matrix: a novel way of transplantation? Hepatology. 2010;52(4):1509-11. 
26. Godoy P, Hewitt NJ, Albrecht U, Andersen ME, Ansari N, Bhattacharya S, et al. Recent advances in 2D and $3 \mathrm{D}$ in vitro systems using primary hepatocytes, alternative hepatocyte sources and non-parenchymal liver cells and their use in investigating mechanisms of hepatotoxicity, cell signaling and ADME. Arch Toxicol. 2013;87(8):1315-530.

27. Lwebuga-Mukasa JS, Thulin G, Madri JA, Barrett C, Warshaw JB. An acellular human amnionic membrane model for in vitro culture of type I pneumocytes: the role of the basement membrane in cell morphology and function. J Cell Physiol. 1984;121(1):215-25

28. Koizumi N, Rigby H, Fullwood NJ, Kawasaki S, Tanioka H, Koizumi K, et al. Comparison of intact and denuded amniotic membrane as a substrate for cell-suspension culture of human limbal epithelial cells. Graefes Arch Clin Exp Ophthalmol. 2007:245(1):123-34.

29. Shortt AJ, Secker GA, Lomas RJ, Wilshaw SP, Kearney JN, Tuft SJ, et al. The effect of amniotic membrane preparation method on its ability to serve as a substrate for the ex-vivo expansion of limbal epithelial cells. Biomaterials. 2009:30(6):1056-65

30. Hsiao YC, Lee HW, Chen YT, Young TH, Yang TL. The impact of compositional topography of amniotic membrane scaffold on tissue morphogenesis of salivary gland. Biomaterials. 2011;32(19):4424-32.

31. Chen YJ, Chung MC, Jane Yao CC, Huang CH, Chang HH, Jeng JH, et al. The effects of acellular amniotic membrane matrix on osteogenic differentiation and ERK1/2 signaling in human dental apical papilla cells. Biomaterials. 2012:33(2):455-63.

32. Berthiaume F, Moghe PV, Toner M, Yarmush ML. Effect of extracellular matrix topology on cell structure, function, and physiological responsiveness: hepatocytes cultured in a sandwich configuration. FASEB J. 1996;10(13):1471-84.

33. Yurchenco PD. Basement membranes: cell scaffoldings and signaling platforms. Cold Spring Harb Perspect Biol. 2011;3(2).

34. Yamasaki CKM, Kato Y, Kakuni M, Usuda S, Ohzone Y, Matsuda S, et al. In vitro evaluation of cytochrome P450 and glucuronidation activities in hepatocytes isolated from liver-humanized mice. Drug Metab Pharmacokinet. 2010;25(6):539-50.

35. Ishizaki TST, Suganuma T, Horai Y, Chiba K, Watanabe M, Asuke W, et al. Pharmacokinetics of ketoprofen following single oral, intramuscular and rectal doses and after repeated oral administration. Eur J Clin Pharmacol. 1980;18(5):407-14

\section{Submit your next manuscript to BioMed Central and take full advantage of:}

- Convenient online submission

- Thorough peer review

- No space constraints or color figure charges

- Immediate publication on acceptance

- Inclusion in PubMed, CAS, Scopus and Google Scholar

- Research which is freely available for redistribution 